\title{
Bifurcations of Travelling Wave Solutions for the $\mathrm{B}(\mathrm{m}, \mathrm{n})$ Equation
}

\author{
Minzhi Wei1 ${ }^{*}$, Yujian Gan1, Shengqiang Tang2 \\ ${ }^{1}$ School of Information and Statistics, Guangxi University of Finance and Economics, Nanning, China \\ ${ }^{2}$ School of Mathematics and Computing Science, Guilin University of Electronic Technology, Guilin, China \\ Email: ${ }^{*}$ weiminzhi@21cn.com
}

Received 22 December 2013; revised 22 January 2014; accepted 29 January 2014

Copyright (C) 2014 by authors and Scientific Research Publishing Inc.

This work is licensed under the Creative Commons Attribution International License (CC BY). http://creativecommons.org/licenses/by/4.0/

(c) (i) Open Access

\begin{abstract}
Using the bifurcation theory of dynamical systems to a class of nonlinear fourth order analogue of the $B(m, n)$ equation, the existence of solitary wave solutions, periodic cusp wave solutions, compactons solutions, and uncountably infinite many smooth wave solutions are obtained. Under different parametric conditions, various sufficient conditions to guarantee the existence of the above solutions are given. Some exact explicit parametric representations of the above waves are determined.
\end{abstract}

\section{Keywords}

Solitary Wave Solution; Periodic Cusp Wave Solution; Periodic Wave Solution; Smoothness of Wave; $B(m, n)$ Equation

\section{Introduction}

Recently, Song and Shao [1] employed bifurcation method of dynamical systems to investigate bifurcation of solitary waves of the following generalized $(2+1)$-dimensional Boussinesq equation

$$
u_{t t}-\alpha u_{x x}-\beta u_{y y}-\gamma\left(u^{2}\right)_{x x}-\delta u_{x x x x}=0
$$

where $\alpha, \beta, \gamma$ and $\delta$ are arbitrary constants with $\gamma \delta \neq 0$. Chen and Zhang [2] obtained some double periodic and multiple soliton solutions of Equation (1.1) by using the generalized Jacobi elliptic function method. Further, Li [3] studied the generalized Boussinesq equation:

$$
\varsigma_{t}+\left[\left(1+\varsigma_{t}\right) u\right]_{x}=a u_{x x x x}
$$

${ }^{*}$ Corresponding author. 
by using bifurcation method. In this paper, we shall employ bifurcation method of dynamical systems [4]-[11] to investigate bifurcation of solitary waves of the following equation:

$$
\left(u^{m}\right)_{t t}=\left(u^{n}\right)_{x x}+\left(u^{n}\right)_{y y}+a\left(u^{2}\right)_{x x}+\delta\left(u^{n}\right)_{x x x x},
$$

Numbers of solitary waves are given for each parameter condition. Under some parameter conditions, exact solitary wave solutions will be obtained. It is very important to consider the dynamical bifurcation behavior for the travelling wave solutions of (1.3). In this paper, we shall study all travelling wave solutions in the parameter space of this system. Let $u(x, y, t)=\phi(x+y-c t)=\phi(\xi)$, where $c$ is the wave speed. Then (1.3) becomes to

$$
c^{2}\left(\phi^{m}\right)^{\prime \prime}=2\left(\phi^{n}\right)^{\prime \prime}+a\left(\phi^{2}\right)^{\prime \prime}+b\left(u^{n}\right)^{(4)},
$$

where "'” is the derivative with respect to $\xi$. Integrating Equation (1.4) twice, using the constants of integration to be zero we find

$$
q \phi^{m}+p \phi^{n}+\phi^{2}+r\left[n(n-1) \phi^{n-2}\left(\phi^{\prime}\right)^{2}+n \phi^{n-1} \phi^{\prime \prime}\right]=0,
$$

where $p=\frac{2}{a}, q=-\frac{c^{2}}{a}, r=\frac{b}{a}$. Equation (1.5) is equivalent to the two-dimensional systems as follows

$$
\frac{\mathrm{d} \phi}{\mathrm{d} \xi}=y, \frac{\mathrm{d} y}{\mathrm{~d} \xi}=-\frac{q \phi^{m}+p \phi^{n}+\phi^{2}+r n(n-1) \phi^{n-2} y^{2}}{r n \phi^{n-1}}
$$

with the first integral

$$
H(\phi, y)=\frac{1}{2} r n \phi^{2(n-1)}+\phi^{n+2}\left[\frac{q}{n+m} \phi^{m-2}+\phi^{2}+\frac{p}{2 n} \phi^{n-2}+\frac{1}{2+n}\right]=h .
$$

System (1.6) is a 5-parameter planar dynamical system depending on the parameter group $(m, n, p, q, r)$. For different $m, n$ and a fixed $r$, we shall investigate the bifurcations of phase portraits of System (1.6) in the phase plane $(\phi, y)$ as the parameters $p, q$ are changed. Here we are considering a physical model where only bounded travelling waves are meaningful. So we only pay attention to the bounded solutions of System (1.6).

\section{Bifurcations of Phase Portraits of (1.6)}

In this section, we study all possible periodic annuluses defined by the vector fields of (1.6) when the parameters $p, q$ are varied.

Let $\mathrm{d} \xi=r n \phi^{n-1} \mathrm{~d} \zeta$, Then, except on the straight lines $\phi=0$, the system (1.6) has the same topological phase portraits as the following system

$$
\frac{\mathrm{d} \phi}{\mathrm{d} \zeta}=r n \phi^{n-1}, \frac{\mathrm{d} y}{\mathrm{~d} \zeta}=-\left[p \phi^{n}+\phi^{2}+r n(n-1) \phi^{n-2} y^{2}\right]
$$

Now, the straight lines $\phi=0$ is an integral invariant straight line of (2.1).

Denote that

$$
f(\phi)=1+q \phi^{m-2}+p \phi^{n-2}, \quad f^{\prime}(\phi)=\phi^{n-3}\left[q(M-2) \phi^{m-n}+p(n-2)\right]
$$

For $m-n=2 l\left(l \in Z^{+}\right), m-1=2 m_{1}-1, n-1=2 n_{1}-1$,

When $\phi=\phi_{0}=\left[-\frac{p(n-2)}{q(m-2)}\right]^{\frac{1}{m-n}}, f^{\prime}\left( \pm \phi_{0}\right)=0$.

We have $f\left( \pm \phi_{0}\right)=1+q\left[-\frac{p(n-2)}{q(m-2)}\right]^{\frac{m-2}{m-n}}+p\left[-\frac{p(n-2)}{q(m-2)}\right]^{\frac{n-2}{m-n}}$

and which imply respectively the relations in the $(p, q)$-parameter plane 


$$
\begin{gathered}
L_{a}: q=-\frac{n-2}{m-2} p^{\frac{m-2}{n-2}}\left(\frac{m-n}{m-2}\right)^{\frac{m-n}{n-2}}, p>0, q<0, \\
L_{b}: q=\frac{n-2}{m-2}(-p)^{\frac{m-2}{n-2}\left(\frac{m-n}{m-2}\right)^{\frac{m-n}{n-2}}, p<0, q>0,}
\end{gathered}
$$

For $m-n=2 l\left(l \in Z^{+}\right), m-1=2 m_{1}, n-1=2 n_{1}-1$, when $\phi=\phi_{0}=\left[-\frac{p(n-2)}{q(m-2)}\right]^{\frac{1}{m-n}}, f^{\prime}\left(\phi_{0}\right)=0$. We have $f\left(\phi_{0}\right)=1+q\left[-\frac{p(n-2)}{q(m-2)}\right]^{\frac{m-2}{m-n}}+p\left[-\frac{p(n-2)}{q(m-2)}\right]^{\frac{n-2}{m-n}}$ and which imply respectively the relations in the $(p, q)$ parameter plane

$$
\begin{gathered}
L_{b}: q=\frac{n-2}{m-2}(-p)^{\frac{m-2}{n-2}}\left(\frac{m-n}{m-2}\right)^{\frac{m-n}{n-2}}, p<0, q>0, \\
L_{c}: q=-\frac{n-2}{m-2}(-p)^{\frac{m-2}{n-2}}\left(\frac{m-n}{m-2}\right)^{\frac{m-n}{n-2}}, p<0, q<0,
\end{gathered}
$$

For $\quad m-n=2 l-1\left(l \in Z^{+}\right), m-1=2 m_{1}-1, n-1=2 n_{1}$, when $\phi=\phi_{0}=\left[-\frac{p(n-2)}{q(m-2)}\right]^{\frac{1}{m-n}}, f^{\prime}\left(\phi_{0}\right)=0$. We have $f\left(\phi_{0}\right)=1+q\left[-\frac{p(n-2)}{q(m-2)}\right]^{\frac{m-2}{m-n}}+p\left[-\frac{p(n-2)}{q(m-2)}\right]^{\frac{n-2}{m-n}}$, which imply respectively the relations in the $(p, q)$ parameter plane

$$
L_{d}: q=-\frac{n-2}{m-2} p^{\frac{m-2}{n-2}}\left(\frac{m-n+4}{m-2}\right)^{\frac{m-n}{n-2}}
$$

For $m-n=2 l-1\left(l \in Z^{+}\right), m-1=2 m_{1}, n-1=2 n_{1}$, when $\phi=\phi_{0}=\left[-\frac{p(n-2)}{q(m-2)}\right]^{\frac{1}{m-n}}, \quad f^{\prime}\left( \pm \phi_{0}\right)=0$. We have $f\left(\phi_{0}\right)=1+q\left[-\frac{p(n-2)}{q(m-2)}\right]^{\frac{m-2}{m-n}}+p\left[-\frac{p(n-2)}{q(m-2)}\right]^{\frac{n-2}{m-n}}$ and $f\left(-\phi_{0}\right)=1-q\left[-\frac{p(n-2)}{q(m-2)}\right]^{\frac{m-2}{m-n}}-p\left[-\frac{p(n-2)}{q(m-2)}\right]^{\frac{n-2}{m-n}}$, which imply respectively the relations in the $(p, q)$-parameter plane

$$
L_{e}: q=-\frac{n-2}{m-2} p^{\frac{m-2}{n-2}}\left(\frac{m-n}{m-2}\right)^{\frac{m-n}{n-2}}, p q<0
$$

Let $M\left(\phi_{e}, y_{e}\right)$ be the coefficient matrix of the linearized system of (2.1) at an equilibrium point $\left(\phi_{e}, y_{e}\right)$. Then, we have

$$
J\left(\phi_{i}, 0\right)=\operatorname{det}\left(M\left(\phi_{e}, 0\right)\right)=r n \phi_{i}^{n-3}\left(q(m-2) \phi_{i}^{m-3}+p(n-2) \phi_{i}^{n-3}\right) .
$$

By the theory of planar dynamical systems, we know that for an equilibrium point of a planar integrable system, if $J<0$ then the equilibrium point is a saddle point; if $J>0$ and $\operatorname{Trace}\left(M\left(\phi_{e}, y_{e}\right)\right)=0$ then it is a center point; if $J>0$ and $\left(\operatorname{Trace}\left(M\left(\phi_{e}, y_{e}\right)\right)\right)^{2}-4 J\left(\phi_{e}, y_{e}\right)>0$, then it is a node; if $J=0$ and the index of the equilibrium point is 0 then it is a cusp, otherwise, it is a high order equilibrium point.

For the function defined by (1.7), we denote that 


$$
h_{i}=H\left(\phi_{i}, 0\right)=\phi_{i}^{n+2}\left[\frac{m-2}{(n-2)(m+n)}+\frac{p(m-n)}{2 n(m+n)} \phi_{i}^{n-2}\right], i=1-4 .
$$

We next use the above statements to consider the bifurcations of the phase portraits of (2.1). In the $(p, q)$ parameter plane, the curves partition it into 4 regions for $m-n=2 l-1, m-n=2 l$ shown in Figure 1 (1-1), (1-2), (1-3), and (1-4), respectively.

1) The case $q \neq 0$, We use Figure 2, Figure 3, Figure 4, and Figure 5 to show the bifurcations of the phase portraits of (2.1).

2) The case $q=0$. We consider the system

$$
\frac{\mathrm{d} \phi}{\mathrm{d} \zeta}=r n \phi^{n-1}, \frac{\mathrm{d} y}{\mathrm{~d} \zeta}=-\left[p \phi^{n}+\phi^{2}+r n(n-1) \phi^{n-2} y^{2}\right]
$$

with the first integral

$$
H(\phi, y)=-\frac{1}{2} r n \phi^{2(n-1)} y^{2}-\phi^{n+2}\left[\frac{p}{2 n} \phi^{n-2}+\frac{1}{2+n}\right]=h .
$$

Figure 6 and Figure 7 show respectively the phase portraits of (2.3) for $n=2 n_{1}$ and $n=2 n_{1}+1$.

\section{Exact Explicit Parametric Representations of Traveling Wave Solutions of (1.6)}

In this section, we give some exact explicit parametric representations of periodic cusp wave solutions.

1). Suppose that $n=4, m=6, r<0,(p, q) \in A_{4}$, In this case, we have the phase portrait of (2.1) shown in Figure 2 (2-5). Corresponding to the orbit defined by $H(\phi, y)=0$ to the equilibrium point $S_{ \pm}\left( \pm \sqrt{\frac{-p+\sqrt{p^{2}-4 q}}{2 q}}, 0\right)$, the arch curve has the algebraic equation

$$
y^{2}=\frac{1}{4(-r)}\left[\phi^{2}-\frac{-\frac{5 p}{4}-5 \sqrt{\frac{p^{2}}{16}-\frac{4 q}{15}}}{2 q}\right]\left[\phi^{2}-\frac{\frac{5 p}{4}-5 \sqrt{\frac{p^{2}}{16}-\frac{4 q}{15}}}{2 q}\right] .
$$

Thus, by using the first Equation of (1.6) and (3.1), we obtain the parametric representation of this arch as follows:

$$
\phi(\xi)= \pm \sqrt{\frac{-\frac{5 p}{4}-5 \sqrt{\frac{p^{2}}{16}-\frac{4 q}{15}}}{2 q}} c n^{-1}\left(\Omega_{1} \xi, k_{1}\right)
$$

where $\Omega_{1}=\sqrt{\frac{5 \sqrt{\frac{p^{2}}{16}-\frac{4 q}{15}}}{4 q r}}, k_{1}=\sqrt{\frac{\frac{p}{4}-\sqrt{\frac{p^{2}}{16}-\frac{4 q}{15}}}{-2 \sqrt{\frac{p^{2}}{16}-\frac{4 q}{15}}}}$.

We will show in Section 4 that (3.10) gives rise to two periodic cusp wave solutions of peak type and valley type of (1.3).

2). Suppose that $n=2, m=4, r>0,(p, q) \in A_{3}$, In this case, we have the phase portrait of (2.1) shown in Figure $2(2-4)$. corresponding to the orbit defined by $H(\phi, y)=0$ to the equilibrium point $A(0,0)$, the arch curve has the algebraic equation

$$
y^{2}=\frac{1}{r} \phi^{2}\left(\frac{q}{6} \phi^{2}+\frac{p+1}{4}\right),
$$

Thus, by using the first equation of (1.6) and (3.3), we obtain the parametric representation of this arch as follows: 
M. Z. Wei et al.
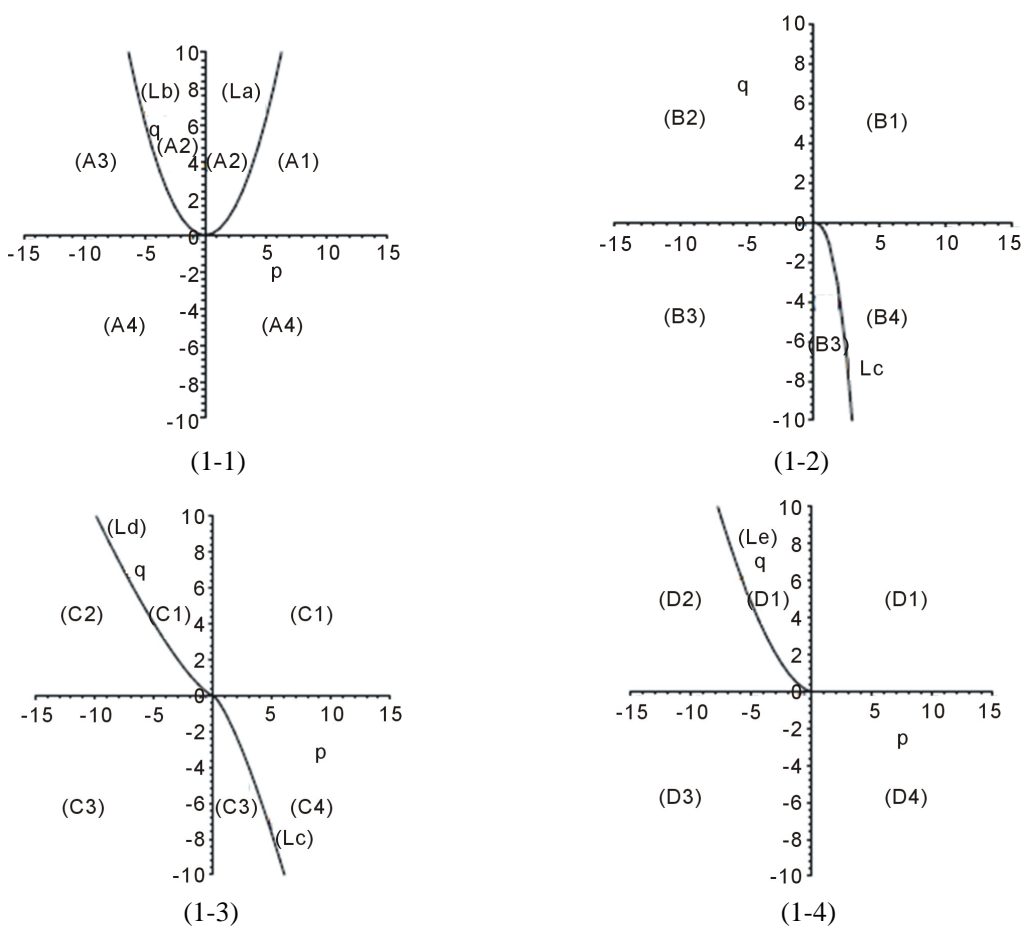

Figure 1. (1-1) $m-n=2 l, n=2 n_{1} ;(1-2) m-n=2 l-1, n=2 n_{1} ;(1-3) m-n=$ $2 \mathrm{l}, \mathrm{n}=2 \mathrm{n}_{1}+1 ;(1-4) \mathrm{m}-\mathrm{n}=2 \mathrm{l}-1, \mathrm{n}=2 \mathrm{n}_{1}+1$.

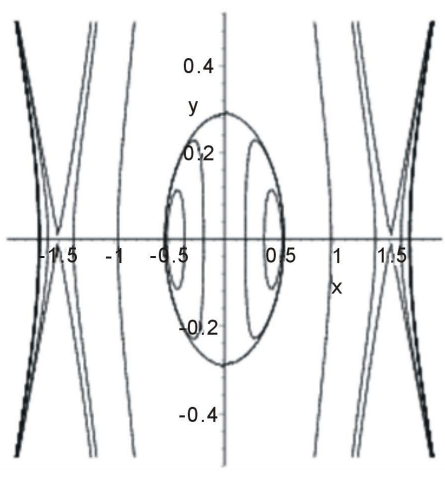

(2-1)

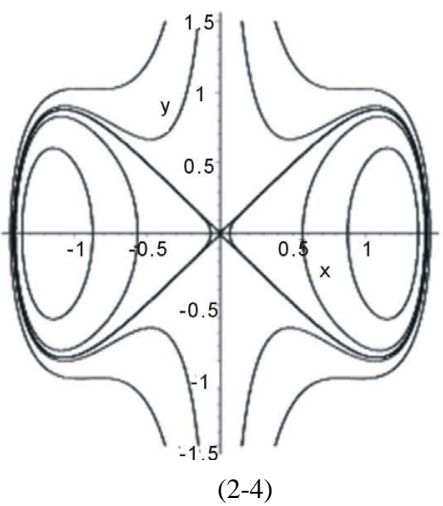

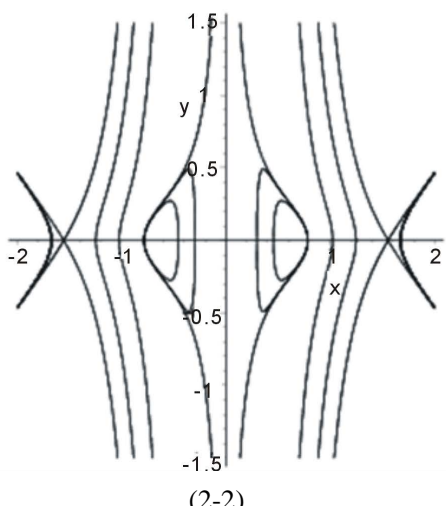

$(2-2)$

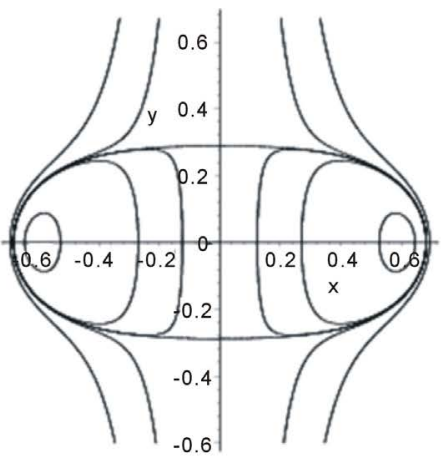

(2-5)

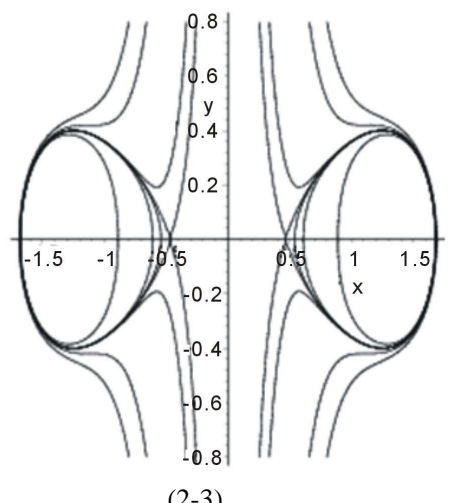

$(2-3)$

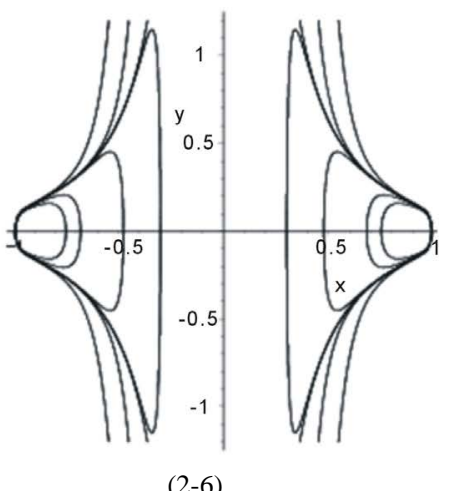

(2-6)

Figure 2. The phase portraits of (1.6) for $\mathrm{m}-\mathrm{n}=2 \mathrm{l}, \mathrm{n}=2 \mathrm{n}_{1}, \mathrm{l}, \mathrm{n}_{1} \in \mathrm{Z}^{+}$. (2-1) $r<0, n_{1}=2,(p, q) \in\left(A_{3}\right)$; (2-2) $r<$ $0, n_{1} \geq 2,(p, q) \in\left(A_{3}\right) ;(2-3) r>0, n_{1} \geq 2,(p, q) \in\left(A_{3}\right) ;(2-4) r>0, n_{1}=1,(p, q) \in\left(A_{3}\right) ;(2-5) r<0, n_{1}=2,(p, q) \in$ $\left(A_{4}\right) ;(2-6) r<0, \mathrm{n}_{1} \geq 3,(p, q) \in\left(A_{3}\right)$.

108 


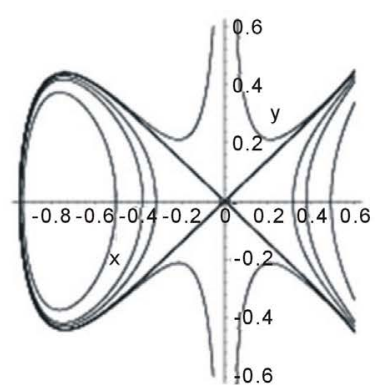

(3-1)

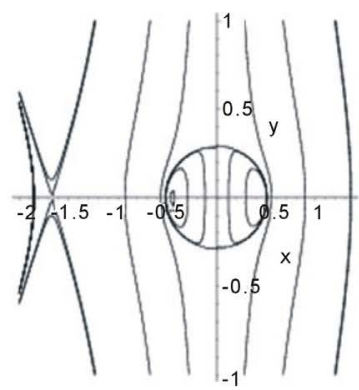

(3-5)

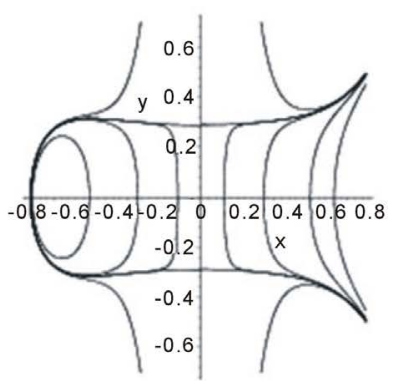

(3-2)

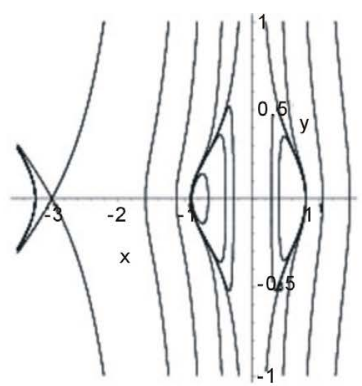

(3-6)

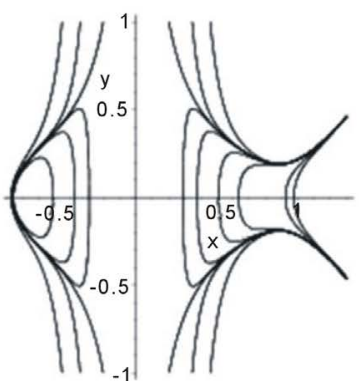

(3-3)

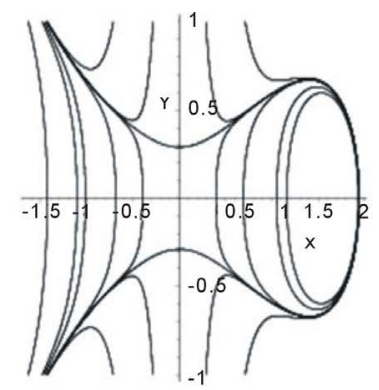

(3-7)

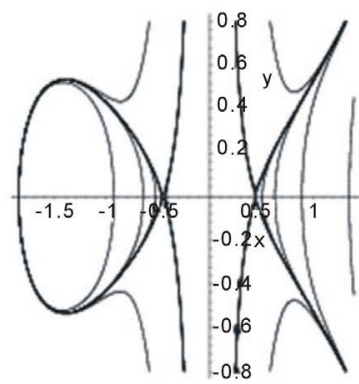

(3-4)

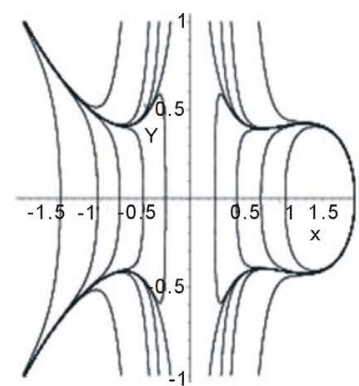

(3-8)

Figure 3. The phase portraits of (1.6) for $\mathrm{m}-\mathrm{n}=2 \mathrm{l}-1, \mathrm{n}=2 \mathrm{n}_{1}, \mathrm{l}, \mathrm{n}_{1} \in \mathrm{Z}^{+}$. (3-1) $r<0, n_{1}=1,(p, q) \in\left(B_{1}\right) ;(3-2) r<0, n_{1}$ $=2,(p, q) \in\left(B_{1} \cup B_{2}\right) ;(3-3) r>0, n_{1} \geq 2,(p, q) \in\left(B_{1}\right) \cup\left(B_{2}\right) ;(3-4) r>0, n_{1} \geq 2,(p, q) \in\left(B_{3}\right) ;(3-5) r<0, n_{1}=2,(p, q)$ $\in\left(B_{3}\right) ;(3-6) r<0, n_{1} \geq 2,(p, q) \in\left(B_{1} \cup B_{2}\right) ;(3-7) r<0, n_{1}=2,(p, q) \in\left(B_{4}\right) \cup\left(B_{2}\right) ;(3-8) r>0, n_{1} \geq 2,(p, q) \in\left(B_{4}\right)$.

$$
\phi(\xi)=\sqrt{\frac{2 q}{3(p+1)}} \sin ^{2}\left(\sqrt{-\frac{q}{6 r}} \xi\right)
$$

We will show in Section 4 that (3.10) gives rise to a solitary wave solutions of peak type and valley type of (1.3).

3). Suppose that $n=3, m=5, r<0,(p, q) \in C_{2}$, In this case, we have the phase portrait of (2.1) shown in Figure 4 (4-5). corresponding to the orbit defined by $H(\phi, y)=0$ to the equilibrium point $A(0,0)$, the arch curve has the algebraic equation

$$
y^{2}=\frac{2}{3(-r)}\left(\phi-\phi_{1}\right)\left(\phi-\phi_{2}\right)\left(\phi_{3}-\phi\right)\left(\phi_{4}-\phi\right)
$$

where $\phi_{1}<\phi_{2}<\phi_{3}<\phi_{4}, \phi_{i}\left(\frac{q}{8} \phi_{i}^{3}+\frac{p}{6} \phi_{i}+\frac{1}{5}\right)=0, i=1-4$.

Thus, by using the first equation of (1.6) and (3.5), we obtain the parametric representation of this arch as follows:

$$
\phi(\xi)=\frac{\left(\phi_{4}-\phi_{1}\right) \phi_{2}-\phi_{1}\left(\phi_{4}-\phi_{2}\right) s n^{2}\left(\Omega_{2} \xi ; k_{2}\right)}{\left(\phi_{4}-\phi_{1}\right)-\left(\phi_{4}-\phi_{2}\right) s n^{2}\left(\Omega_{2} \xi ; k_{2}\right)}
$$

where $\operatorname{sn}(x ; k)$ is the Jacobin elliptic functions with the modulo $k$,

$$
\Omega_{2}=\sqrt{\frac{\left(\phi_{3}-\phi_{2}\right)\left(\phi_{3}-\phi_{1}\right)}{-6 r}}, \quad k_{2}=\sqrt{\frac{\left(\phi_{3}-\phi_{2}\right)\left(\phi_{4}-\phi_{1}\right)}{\left(\phi_{4}-\phi_{2}\right)\left(\phi_{3}-\phi_{1}\right)}},
$$

We will show in Section 4 that (3.6) gives rise to a smooth compacton solution of (1.3).

4). Suppose that $n=2, m=3, r<0,(p, q) \in B_{1}$. In this case, we have the phase portrait of (2.1) shown in Figure 3 (3-1), corresponding to the orbit defined by $H(\phi, y)=0$ to the equilibrium point $A(0,0)$, the arch curve has the algebraic equation 


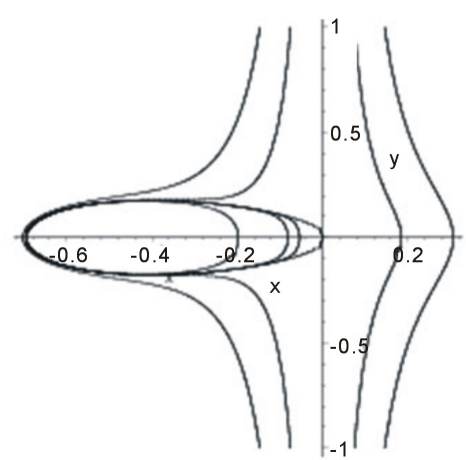

(4-1)

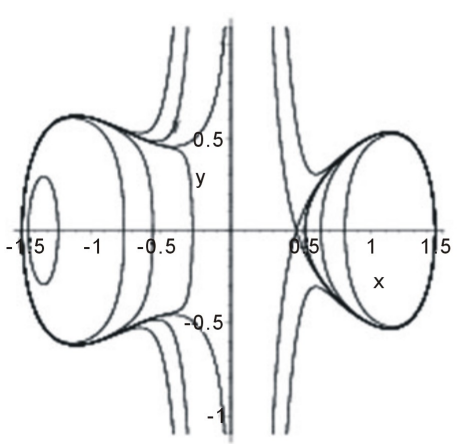

(4-4)

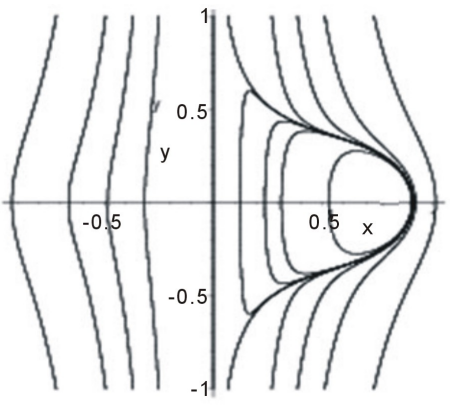

(4-7)

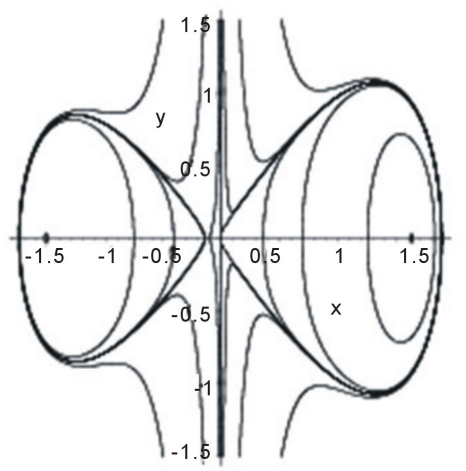

(4-10)

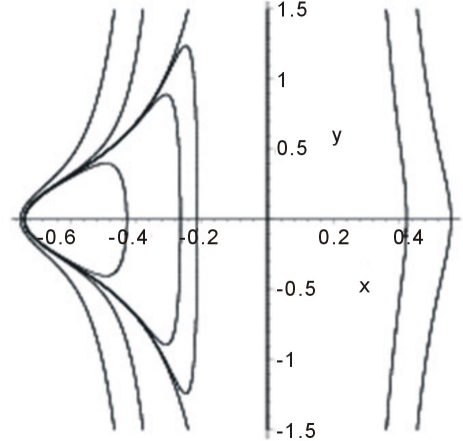

(4-2)

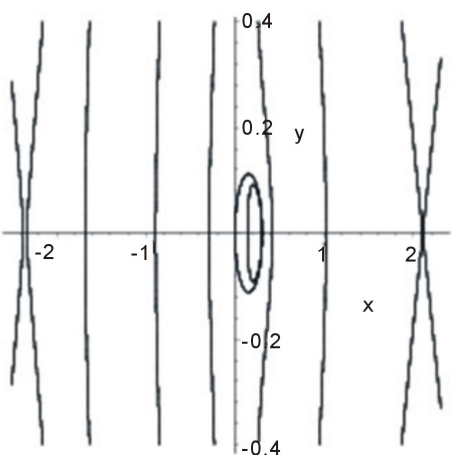

(4-5)

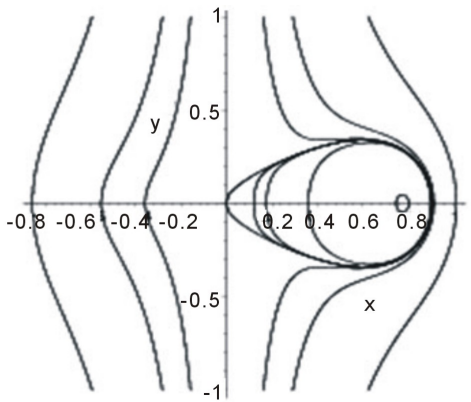

(4-8)

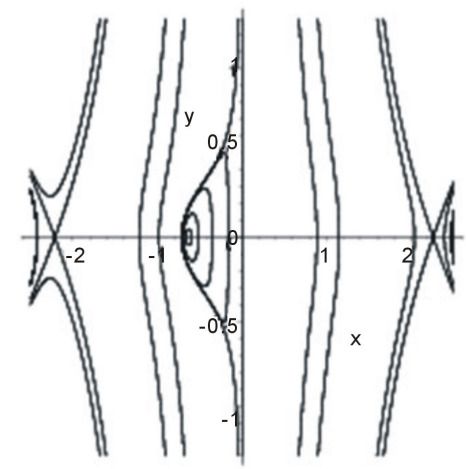

(4-11)

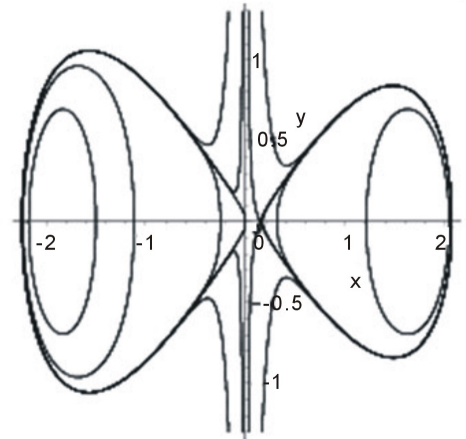

(4-3)

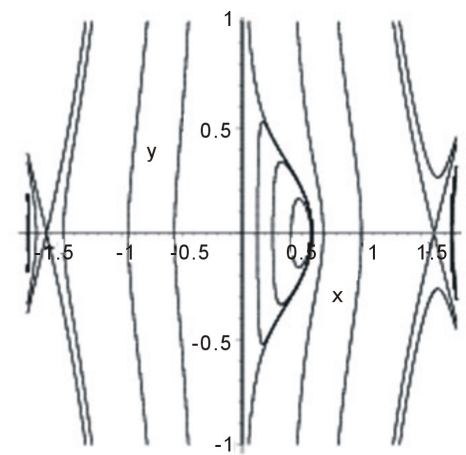

(4-6)

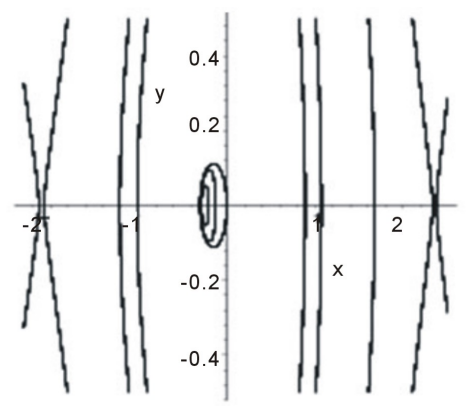

(4-9)

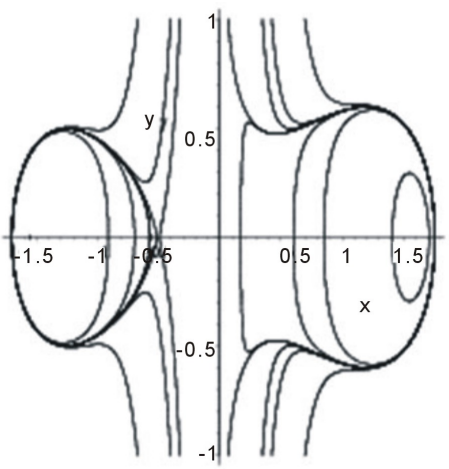

(4-12)

Figure 4. (4-1) $r>0, n_{1}=1,(p, q) \in\left(C_{1}\right)$; (4-2) $r>0, n_{1} \geq 1,(p, q) \in\left(C_{2}\right) ;(4-3) r>0, n_{1}=1,(p, q) \in\left(C_{2}\right)$; (4-4) $r>0, n_{1} \geq 2,(p, q) \in\left(C_{2}\right) ;(4-5) r<0, n_{1}=1,(p, q) \in\left(C_{2}\right) ;(4-6) r<0, n_{1} \geq 2,(p, q) \in\left(C_{2}\right) ;(4-7) r>0, n_{1} \geq 2,(p$, q) $\in\left(C_{3}\right)$; (4-8) $r<0, n_{1}=1,(p, q) \in\left(C_{3}\right)$; (4-9) $r>0, n_{1}=1,(p, q) \in\left(C_{4}\right) ;(4-10) r<0, n_{1}=1,(p, q) \in\left(C_{4}\right)$; (4-11) $r>0, n_{1} \geq 2,(p, q) \in\left(C_{4}\right) ;(4-12) r<0, n_{1} \geq 2,(p, q) \in\left(C_{4}\right)$. 


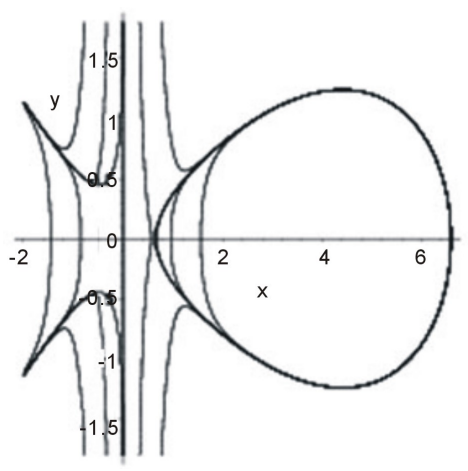

(5-1)

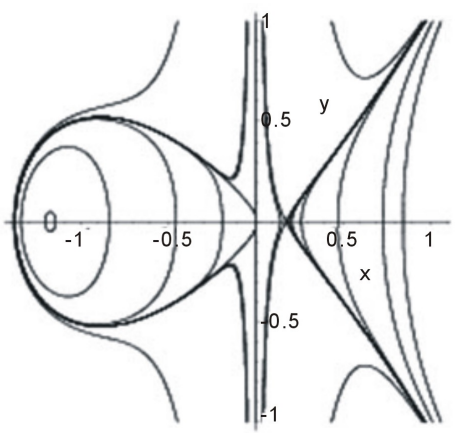

(5-4)

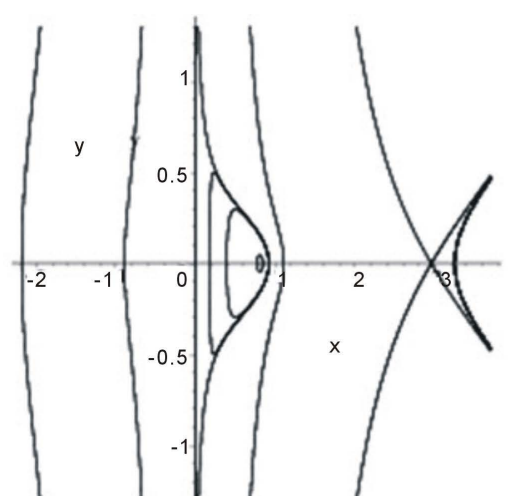

(5-7)

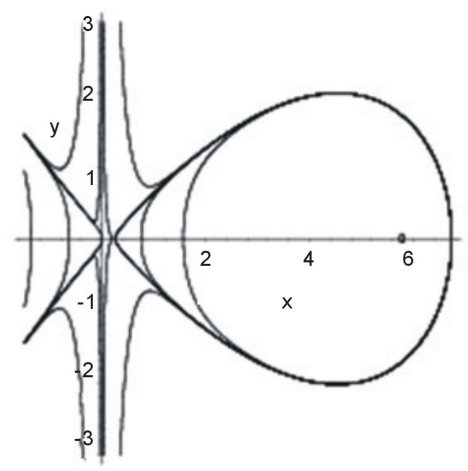

(5-2)

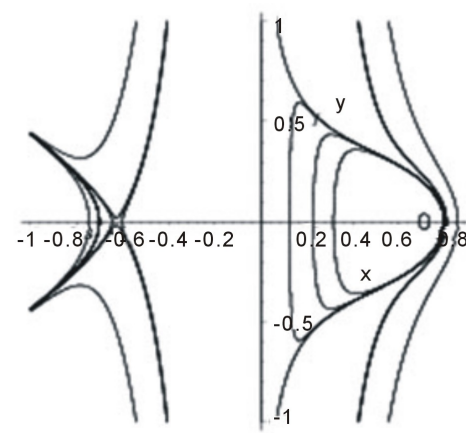

(5-5)

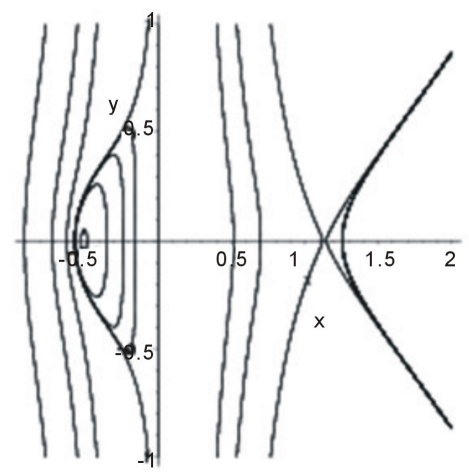

(5-3)

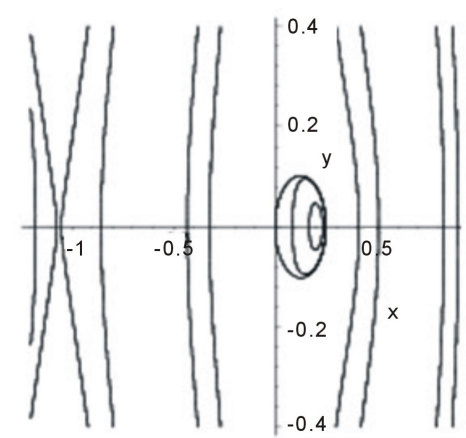

(5-6)

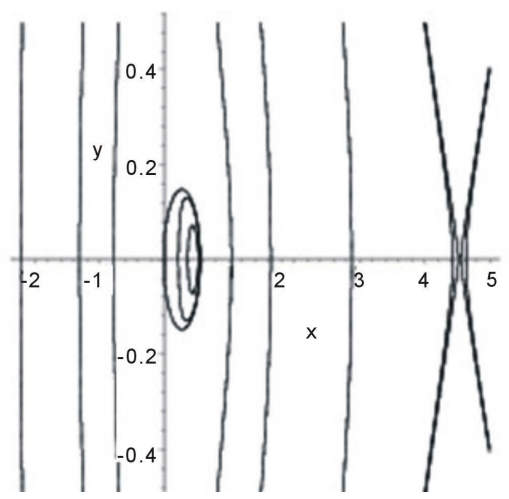

(5-8)

Figure 5. The phase portraits of (1.6) for $\mathrm{m}-\mathrm{n}=2 \mathrm{l}-1, \mathrm{n}=2 \mathrm{n}_{1}+1, \mathrm{l}, \mathrm{n}_{1} \in \mathrm{Z}^{+}(5-1) r>0, n_{1} \geq 2,(p, q) \in\left(D_{2}\right)$; (5-2) $r>0, n_{1}=1,(p, q) \in\left(D_{2}\right) ;(5-3) r>0, n_{1} \geq 2,(p, q) \in\left(D_{3}\right) \cup\left(D_{4}\right) ;(5-4) r>0, n_{1}=1,(p, q) \in\left(D_{3}\right) \cup\left(D_{4}\right) ;(5-5)$ $r<0, n_{1} \geq 2,(p, q) \in\left(D_{3}\right) \cup\left(D_{4}\right) ;(5-6) r<0, n_{1}=1,(p, q) \in\left(D_{3}\right) \cup\left(D_{4}\right) ;(5-7) r<0, n_{1} \geq 2,(p, q) \in\left(D_{2}\right) ;(5-8) r<$ $0, n_{1}=1,(p, q) \in\left(D_{2}\right)$.

$$
y^{2}=\frac{q}{-5 r} \phi^{2}\left(\phi+\frac{5(p+1)}{4 q}\right),
$$

Thus, by using the first equation of (1.6) and (3.7), we obtain the parametric representation of this arch as follows:

$$
\phi(\xi)=\frac{-\frac{5(p+1)}{4 q}}{1-\tanh ^{2}\left(\sqrt{-\frac{4 q}{5 r}} \xi\right)} .
$$




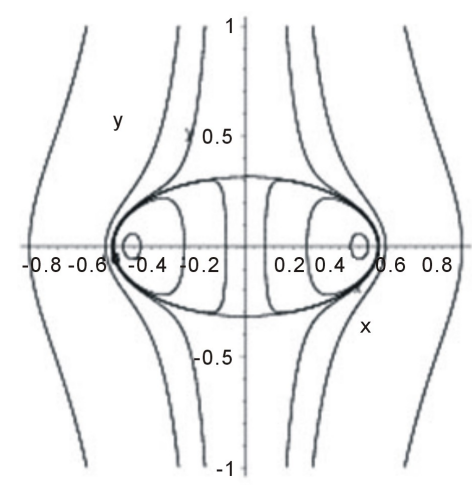

$(6-1)$

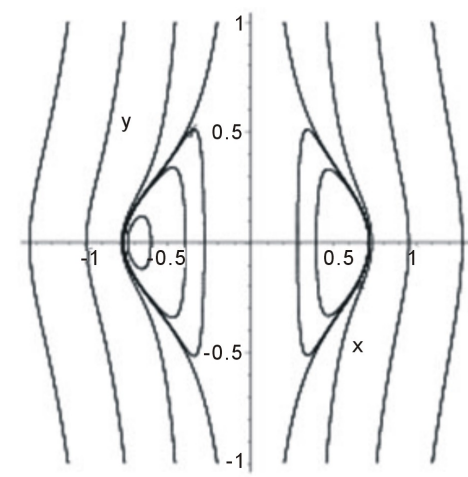

$(6-2)$

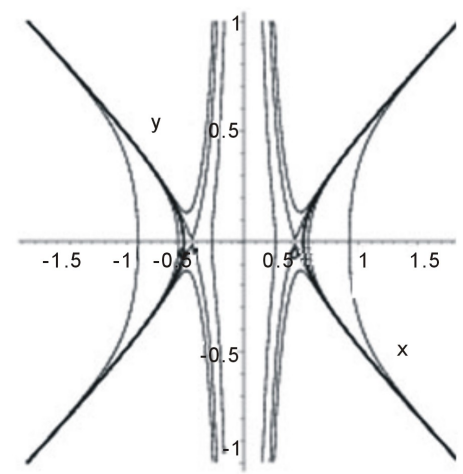

$(6-3)$

Figure 6. The phase portraits of (1.6) for $\mathrm{n}=2 \mathrm{n}_{1}, \mathrm{n}_{1} \in \mathrm{Z}^{+}$. (6-1) $r<0, n_{1}=2, \mathrm{~m}_{1} \geq \mathrm{n}_{1}, p<0$; (6-2) $r<0, n_{1} \geq 2, \mathrm{~m}_{1}>\mathrm{n}_{1}$, $p<0$; (6-3) $r>0, \mathrm{~m}_{1}>\mathrm{n}_{1}, p<0$.

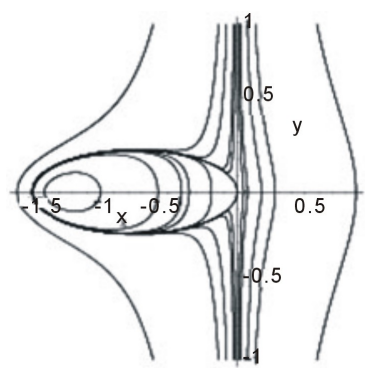

$(7-1)$

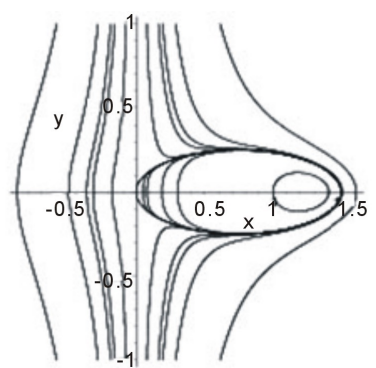

$(7-2)$

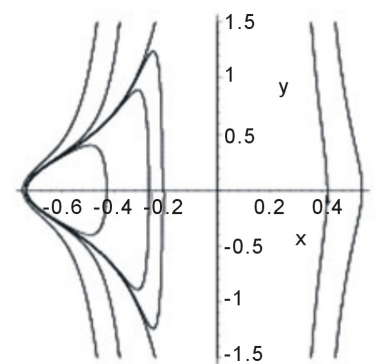

$(7-3)$

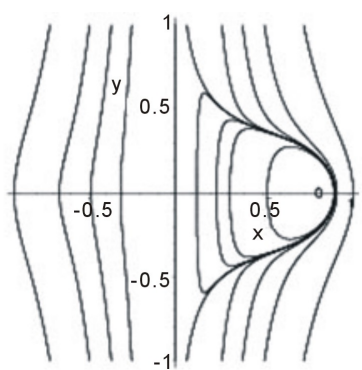

$(7-4)$

Figure 7. The phase portraits of (1.6) for $\mathrm{n}=2 \mathrm{n}_{1}+1, \mathrm{n}_{1} \in \mathrm{Z}^{+}$. (7-1) $r>0, n_{1}=1, \mathrm{~m}_{1} \geq \mathrm{n}_{1}, p>0$; (7-2) $r<0, n_{1}=1, \mathrm{~m}_{1}>\mathrm{n}_{1}, p$ $<0$; (7-3) $r<0, n_{1} \geq 2, \mathrm{~m}_{1}>\mathrm{n}_{1}, p>0,(7-4) r<0, n_{1} \geq 2, \mathrm{~m}_{1}>\mathrm{n}_{1}, p<0$.

We will show in Section 4 that (3.8) gives rise to a solitary wave solution of peak type or valley type of (1.3).

5). Suppose that $n=3, m=4, r>0,(p, q) \in D_{3} \cup D_{4}$,in this case, we have the phase portrait of (2.1) shown in Figure 5 (5-4). corresponding to the orbit defined by $H(\phi, y)=0$ to the equilibrium point

$S_{ \pm}\left( \pm \sqrt{\frac{-p+\sqrt{p^{2}-4 q}}{2 q}}, 0\right)$, the arch curve has the algebraic equation

$$
y^{2}=\frac{2}{3 r}(\phi-0)\left(\phi-\left(-\frac{p}{12}+\frac{7}{2 q} \sqrt{\frac{p^{2}}{36}-\frac{4 q}{35}}\right)\right)\left(-\frac{p}{12}-\frac{7}{2 q} \sqrt{\frac{p^{2}}{36}-\frac{4 q}{35}-\phi}\right) .
$$

Thus, by using the first equation of (1.6) and (3.9), we obtain the parametric representation of this arch as follows:

$$
\phi(\xi)=-\frac{7 p}{12 q}-\frac{7}{2 q} \sqrt{\frac{p^{2}}{36}-\frac{4 q}{35}}+\left(\frac{7 p}{12 q}+\frac{7}{2 q} \sqrt{\frac{p^{2}}{36}-\frac{4 q}{35}}\right) \operatorname{sn}^{2}\left(\Omega_{3} \xi ; k_{3}\right),
$$

where $\operatorname{sn}(x ; k)$ is the Jacobin elliptic functions with the modulo $k$ and

$$
\Omega_{3}=\sqrt{\frac{-7 \sqrt{\frac{p^{2}}{36}-\frac{4 q}{35}}}{6 q r}}, \quad k_{3}=\sqrt{\frac{1}{12 \sqrt{\frac{p^{2}}{36}-\frac{4 q}{35}}}+\frac{1}{2}},
$$

We will show in Section 4 that (3.10) gives rise to a smooth compacton solution of (1.3). 
6). Suppose that $n=3, m=4, r<0,(p, q) \in D_{3} \cup D_{4}$, In this case, we have the phase portrait of (2.1) shown in Figure 5 (5-6), corresponding to the orbit defined by $H(\phi, y)=0$ to the equilibrium point

$S_{ \pm}\left( \pm \sqrt{\frac{-p \pm \sqrt{p^{2}-4 q}}{2 q}}, 0\right)$, the arch curve has the algebraic equation

$$
y^{2}=\frac{2}{-3 r}(0-\phi)\left(\phi-\left(-\frac{p}{12}+\frac{7}{2 q} \sqrt{\frac{p^{2}}{36}-\frac{4 q}{35}}\right)\right)\left(-\frac{p}{12}-\frac{7}{2 q} \sqrt{\frac{p^{2}}{36}-\frac{4 q}{35}-\phi}\right) .
$$

Thus, by using the first equation of (1.6) and (3.11), we obtain the parametric representation of this arch as follows:

$$
\phi(\xi)=-\frac{7 p}{12 q}-\frac{7}{2 q} \sqrt{\frac{p^{2}}{36}-\frac{4 q}{35}}+\frac{\frac{7}{q} \sqrt{\frac{p^{2}}{36}-\frac{4 q}{35}}\left(\frac{7 p}{12 q}-\frac{7}{2 q} \sqrt{\frac{p^{2}}{36}-\frac{4 q}{35}}\right)}{\left(\frac{7 p}{12 q}+\frac{7}{2 q} \sqrt{\frac{p^{2}}{36}-\frac{4 q}{35}}\right) \operatorname{sn}^{2}\left(\Omega_{4} \xi ; k_{4}\right)},
$$

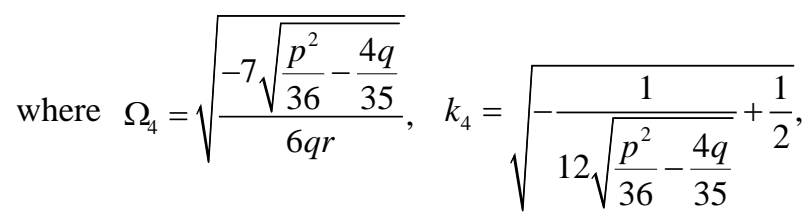

we will show in Section 4 that (3.20) gives rise to a smooth compacton solution of (1.3)

7). Suppose $n=4, m=5, r<0,(p, q) \in B_{1} \cup B_{2} \cup B_{4}$ that. In this case, we have the phase portrait of (2.1) shown in Figure 3 (3-2) and (3-7), corresponding to the orbit defined by $H(\phi, y)=0$ to the equilibrium point $A(0,0)$, the arch curve has the algebraic equation

$$
y^{2}=\frac{1}{-2 r}\left(\frac{q}{9} \phi^{3}+\frac{p}{8} \phi^{2}+\frac{1}{6}\right)
$$

Thus, by using the first equation of (1.6) and (3.13), we obtain the parametric representation of this arch as follows:

$$
\phi(\xi)=\frac{3 p}{-8 q}+\wp\left(\sqrt{-\frac{q}{72 r}} \xi, g_{1}, g_{2}\right)
$$

where $g_{1}=\frac{27 p^{2}}{16 q^{2}}, g_{2}=\frac{27 p^{3}}{128 q^{3}}-\frac{6}{q}$. We will show in Section 4 that (3.14) gives rise to a smooth compacton solution of (1.3).

8). Suppose $n=4, m=6, r<0,(p, q) \in A_{3}$. In this case, we have the phase portrait of (2.1) shown in Figure 2 (2-1), corresponding to the orbit defined by $H(\phi, y)=0$ to the equilibrium point $A(0,0)$, the arch curve has the algebraic equation

$$
y^{2}=\frac{1}{-4 r}\left(\phi^{2}+\frac{5 p}{8 q}+\frac{5}{2 q} \sqrt{\frac{p^{2}}{16}-\frac{4 q}{15}}\right)\left(\phi^{2}+\frac{5 p}{8 q}-\frac{5}{2 q} \sqrt{\frac{p^{2}}{16}-\frac{4 q}{15}}\right) .
$$

Thus, by using the first equation of (1.6) and (3.15), we obtain the parametric representation of this arch as follows:

$$
\phi(\xi)= \pm \frac{\sqrt{-\frac{5 p}{8 q}+\frac{5}{2 q} \sqrt{\frac{p^{2}}{16}-\frac{4 q}{15}}}}{\operatorname{cn}\left(\Omega_{5} \xi ; k_{5}\right)},
$$


where $\Omega_{5}=\sqrt{\frac{5 \sqrt{\frac{p^{2}}{16}-\frac{4 q}{15}}}{-4 q r}}, \quad k_{4}=\sqrt{\frac{p}{8 \sqrt{\frac{p^{2}}{16}-\frac{4 q}{15}}}+\frac{1}{2}}$,

We will show in Section 4 that (3.16) gives rise to two periodic cusp wave solutions of peak type and valley type of (1.3).

9). Suppose $n=3, m=5, r>0,(p, q) \in C_{4}$. In this case, we have the phase portrait of (2.1) shown in Figure 4 (4-5), corresponding to the orbit defined by $H(\phi, y)=0$ to the equilibrium point $A(0,0)$, the arch curve has the algebraic equation

$$
y^{2}=\frac{2}{3 r}\left(\phi-\phi_{1}\right)\left(\phi-\phi_{2}\right)\left(\phi-\phi_{3}\right)\left(\phi_{4}-\phi\right)
$$

where $\phi_{1}<\phi_{2}<\phi_{3}<\phi_{4}, \phi_{i}\left(-\frac{q}{8} \phi_{i}^{3}-\frac{p}{6} \phi_{i}-\frac{1}{5}\right)=0, i=1-4$. Thus, by using the first equation of (1.6) and (3.17), we obtain the parametric representation of this arch as follows:

$$
\phi(\xi)=\frac{\left(\phi_{4}-\phi_{3}\right) \phi_{2}-\phi_{3}\left(\phi_{4}-\phi_{2}\right) s n^{2}\left(\Omega_{6} \xi ; k_{6}\right)}{\left(\phi_{4}-\phi_{2}\right)-\left(\phi_{4}-\phi_{3}\right) s n^{2}\left(\Omega_{6} \xi ; k_{6}\right)},
$$

where $\operatorname{sn}(x ; k)$ is the Jacobin elliptic functions with the modulo $k$ and

$$
\Omega_{6}=\sqrt{\frac{\left(\phi_{4}-\phi_{2}\right)\left(\phi_{3}-\phi_{1}\right)}{6 r}}, \quad k_{6}=\sqrt{\frac{\left(\phi_{4}-\phi_{3}\right)\left(\phi_{2}-\phi_{1}\right)}{\left(\phi_{3}-\phi_{2}\right)\left(\phi_{3}-\phi_{1}\right)}},
$$

We will show in Section 4 that (3.6) gives rise to a smooth compacton solution of (1.3).

\section{The Existence of Smooth and Non-Smooth Travelling Wave Solutions of (1.6)}

In this section, we use the results of Section 2 to discuss the existence of smooth and non-smooth solitary wave and periodic wave solutions. We first consider the existence of smooth solitary wave solution and periodic wave solutions.

\section{Theorem 4.1}

1). Suppose that $m-n=2, n=2 n_{1}+1 \geq 5, l, n_{1} \in Z^{+}, r>0,(p, q) \in C_{2}$ : Then, corresponding to a branch of the curves $H(\phi, y)=h_{2}\left(h_{3}\right)$ defined by (1.7), Equation (1.3) has a smooth solitary wave solution of peak type, corresponding to a branch of the curves $H(\phi, y)=h, h \in\left(h_{3}, h_{2}\right)$ defined by (1.7), Equation (1.3) has a smooth family of periodic wave solutions (see Figure 4 (4-4)).

2). Suppose that $m-n=2, n=3, m \in Z^{+}, r>0,(p, q) \in C_{2}$ : Then, corresponding to a branch of the curves $H(\phi, y)=h_{2}$ defined by (1.7), equation (1.3) has a smooth solitary wave solution of peak type, corresponding to a branch of the curves $H(\phi, y)=h, h \in\left(h_{3}, h_{2}\right)$ defined by (1.7), Equation (1.3) has a smooth family of periodic wave solutions (see Figure 4 (4-3)).

3). Suppose that $m-n=2, n=2 n_{1}+1 \geq 5, l, n_{1} \in Z^{+}, r>0,(p, q) \in A_{2}$, Then, corresponding to a branch of the curves $H(\phi, y)=0$ defined by (1.7), equation (1.3) has a smooth solitary wave solution of peak type, corresponding to a branch of the curves $H(\phi, y)=h, h \in\left(h_{1}, h_{2}\right)$ defined by (1.7), equation (1.3) has a smooth family of periodic wave solutions (see Figure 4 (4-12)).

4). Suppose that $m-n=2 l, n=3, m \in Z^{+}, r>0,(p, q) \in C_{2}$, Then, corresponding to a branch of the curves $H(\phi, y)=0$ defined by (1.7), equation (1.3) has a smooth solitary wave solution of valley type, corresponding to a branch of the curves $H(\phi, y)=h, h \in\left(h_{1}, h_{3}\right)$ defined by (1.7), equation (1.3) has a smooth family of periodic wave solutions (see Figure 4 (4-3)). 
5). Suppose that $m-n=2 l, n \geq 4, m \in Z^{+}, r>0,(p, q) \in A_{2}$, Then, corresponding to a branch of the curves $H(\phi, y)=h_{2}$ defined by (1.7), equation (1.3) has a smooth solitary wave solution of peak type, corresponding to a branch of the curves $H(\phi, y)=h, h \in\left(h_{3}, h_{4}\right)$ defined by (1.7), Equation (1.3) has a smooth family of periodic wave solutions (see Figure 2 (2-3)).

6). Suppose that $m-n=2 l, n \geq 4, m \in Z^{+}, r>0,(p, q) \in A_{2}$, Then, corresponding to a branch of the curves $H(\phi, y)=h_{3}$ defined by (1.7), equation (1.3) has a smooth solitary wave solution of peak type, corresponding to a branch of the curves $H(\phi, y)=h, h \in\left(h_{1}, h_{2}\right)$ defined by (1.7), Equation (1.3) has a smooth family of periodic wave solutions (see Figure 2 (2-3)).

7). Suppose that $m-n=2 l, n=3, m \in Z^{+}, r>0,(p, q) \in C_{3}$, Then, corresponding to a branch of the curves $H(\phi, y)=0$ defined by (1.7), Equation (1.3) has a smooth solitary wave solution of valley type, corresponding to a branch of the curves $H(\phi, y)=h, h \in\left(h_{1}, h_{2}\right)$ defined by (1.7), Equation (1.3) has a smooth family of periodic wave solutions (see Figure 2 (2-3)).

8). Suppose that $m-n=2 l-1, n=4, l \in Z^{+}, r>0,(p, q) \in B_{3}$, Then, corresponding to a branch of the curves $H(\phi, y)=h, h \in\left(h_{3}, h_{2}\right)$ defined by (1.7), Equation (1.3) has a smooth family of periodic wave solutions (see Figure 3 (3-5)).

9). Suppose that: $m-n=2 l-1, n \geq 4, l \in Z^{+}, r>0,(p, q) \in B_{3}$, Then, corresponding to a branch of the curves $H(\phi, y)=h_{2}$ defined by (1.7), equation (1.3) has a smooth solitary wave solution of valley type, corresponding to a branch of the curves $H(\phi, y)=h, h \in\left(h_{1}, h_{2}\right)$ defined by (1.7), Equation (1.3) has a smooth family of periodic wave solutions (see Figure 3 (3-4)).

10). Suppose that $m-n=2 l, n=2, l \in Z^{+}, r<0,(p, q) \in B_{1}$, Then, corresponding to a branch of the curves $H(\phi, y)=0$ defined by (1.7), equation (1.3) has a smooth solitary wave solution of valley type, corresponding to a branch of the curves $H(\phi, y)=h, h \in\left(0, h_{1}\right)$ defined by (1.7), Equation (1.3) has a smooth family of periodic wave solutions (see Figure 2 (2-4)).

11). Suppose that $m-n=2 l-1, n=2, l \in Z^{+}, r<0,(p, q) \in B_{1}$ : Then, corresponding to a branch of the curves $H(\phi, y)=0$ defined by (1.7), Equation (1.3) has a smooth solitary wave solution of valley type, corresponding to a branch of the curves $H(\phi, y)=h, h \in\left(0, h_{1}\right)$ defined by (1.7), Equation (1.3) has a smooth family of periodic wave solutions (see Figure 3 (3-1)).

We shall describe what types of non-smooth solitary wave and periodic wave solutions can appear for our system (1.6) which correspond to some orbits of (2.1) near the straight line $\phi=0$. To discuss the existence of cusp waves, we need to use the following lemmarelating to the singular straight line.

Lemma 4.2 The boundary curves of a periodic annulus are the limit curves of closed orbits inside the annulus; If these boundary curves contain a segment of the singular straight line $\phi=0$ of (1.4), then along this segment and near this segment, in very short time interval $y=\phi_{\xi}$ jumps rapidly.

Base on Lemma 4.2, Figure 2, and Figure 3, we have the following result.

\section{Theorem 4.3}

1). Suppose that $m-n=2 l, n=4, l \in Z^{+}$.

a). For $r<0,(p, q) \in A_{4}$ corresponding to the arch curve $H(\phi, y)=0$ defined by (1.7), Equation (1.3) has two periodic cusp wave solutions; corresponding to two branches of the curves $H(\phi, y)=h, h \in\left(h_{1}, 0\right)$ defined by (1.7), Equation (1.3) has two families of periodic wave solutions. When h varies from $h_{1}$ to 0 , these periodic travelling waves will gradually lose their smoothness, and evolve from smooth periodic travelling waves to periodic cusp travelling waves, finally approach a periodic cusp wave of valley type and a periodic cusp wave of peak type defined by $H(\phi, y)=0$ of (1.7) (see Figure 2 (2-5)). 
b). For $r>0,(p, q) \in A_{3}$ corresponding to the arch curve $H(\phi, y)=h, h \in\left(0, h_{2}\right)$ defined by (1.7), Equation (1.3) has two periodic cusp wave solutions; corresponding to two branches of the curves $H(\phi, y)=h, h \in\left(h_{1}, 0\right)$ defined by (1.7), Equation (1.3) has two families of periodic wave solutions. When h varies from $h_{1}$ to 0 , these periodic travelling waves will gradually lose their smoothness, and evolve from smooth periodic travelling waves to periodic cusp travelling waves, finally approach a periodic cusp wave of valley type and a periodic cusp wave of peak type defined by $H(\phi, y)=0$ of (1.7) (see Figure 2 (2-1)).

2). Suppose that $m-n=2 l-1, n=4, l \in Z^{+}$.

c). For $r<0,(p, q) \in B_{4}$ corresponding to the arch curve $H(\phi, y)=0$ defined by (1.7), Equation (1.3) has two periodic cusp wave solutions; corresponding to two branches of the curves $H(\phi, y)=h, h \in\left(0, h_{1}\right)$ defined by (1.7), Equation (1.3) has two families of periodic wave solutions. When h varies from 0 to $h_{1}$, these periodic travelling waves will gradually lose their smoothness, and evolve from smooth periodic travelling waves to periodic cusp travelling waves, finally approach a periodic cusp wave of valley type and a periodic cusp wave of peak type defined by $H(\phi, y)=0$ of (1.7) (see Figure 3 (3-7)).

d). For $r<0,(p, q) \in B_{3}$ corresponding to the arch curve $H(\phi, y)=0$ defined by (1.7), Equation (1.3) has two periodic cusp wave solutions; corresponding to two branches of the curves $H(\phi, y)=h, h \in\left(0, h_{3}\right)$ defined by (1.7), Equation (1.3) has two families of periodic wave solutions. When h varies from 0 to $h_{3}$, these periodic travelling waves will gradually lose their smoothness, and evolve from smooth periodic travelling waves to periodic cusp travelling waves, finally approach a periodic cusp wave of valley type and a periodic cusp wave of peak type defined by $H(\phi, y)=0$ of (1.7) (see Figure 3 (3-5)).

d). For $r<0,(p, q) \in B_{1} \cup B_{2}$ corresponding to the arch curve $H(\phi, y)=0$ defined by (1.7), Equation (1.3) has two periodic cusp wave solutions; corresponding to two branches of the curves $H(\phi, y)=h, h \in\left(0, h_{1}\right)$ defined by (1.7), Equation (1.3) has two families of periodic wave solutions. When h varies from 0 to $h_{1}$, these periodic travelling waves will gradually lose their smoothness, and evolve from smooth periodic travelling waves to periodic cusp travelling waves, finally approach a periodic cusp wave of valley type and a periodic cusp wave of peak type defined by $H(\phi, y)=0$ of (1.7) (see Figure 3 (3-3)).

We can easily see that there exist two families of closed orbits of (1.3) in Figure 2 (2-6), Figure 3 (3-6) and in Figure 6 (6-2). There is one family of closed orbits in Figure 3 (3-3), (3-8), Figure 4 (4-2), (4-5) - (4-7), (4-9), (4-11) and in Figure 5 (5-3), (5-5) - (5-7) and in Figure 7 (7-3), (7-4). In all the above cases there exists at least one family of closed orbits (1.3) for which as whichh from $H\left(\phi_{e}, 0\right)$ to 0 , where $\phi_{e}$ is the abscissa of the center, the closed orbit will expand outwards to approach the straight line $\phi=0$ and $|y|=\left|\phi^{\prime}\right|$ will approach to $\infty$. As a result, we have the following conclusions.

\section{Theorem 4.4}

1). Suppose that $m-n=2 l, n=2 n_{1}+1, l, n_{1} \in Z^{+}$.

a). If $r<0, n_{1} \geq 1,(p, q) \in C_{2}$; then when $h \in\left(h_{1}, 0\right)$ in (1.7), Equation (1.3) has a family of uncountably infinite many periodic traveling wave solutions; where $h$ varies from $h_{1}$ to 0 , these periodic traveling wave solutions will gradually lose their smoothness, and evolve from smooth periodic traveling waves to periodic cusp traveling waves (see Figure 4 (4-2)).

b). If $r<0, n_{1} \geq 2,(p, q) \in C_{3}$; then when $h \in\left(0, h_{1}\right)$ in (1.7), Equation (1.3) has a family of uncountably infinite many periodic traveling wave solutions; when $h$ varies from 0 to $h_{1}$, these periodic traveling wave solutions will gradually lose their smoothness, and evolve from smooth periodic traveling waves to periodic cusp traveling waves (see Figure 4 (4-7)).

c). If $r<0, n_{1} \geq 2,(p, q) \in C_{2}$; then when $h \in\left(0, h_{2}\right)$ in (1.7), Equation (1.3) has a family of uncountably infinite many periodic traveling wave solutions; when $h$ varies from 0 to $h_{2}$, these periodic traveling wave solutions will gradually lose their smoothness, and evolve from smooth periodic traveling waves to periodic cusp traveling waves (see Figure 4 (4-6)). 
d). If $r>0, n_{1} \geq 2,(p, q) \in C_{4}$; then when $h \in\left(h_{2}, 0\right)$ in (1.7), Equation (1.3) has a family of uncountably infinite many periodic traveling wave solutions; when $h$ varies from $h_{2}$ to 0 , these periodic traveling wave solutions will gradually lose their smoothness, and evolve from smooth periodic traveling waves to periodic cusp traveling waves (see Figure 4 (4-11)).

e). If $r<0, n_{1}=1,(p, q) \in C_{2}$; then when $h \in\left(0, h_{2}\right)$ in (1.7), Equation (1.3) has a family of uncountably infinite many periodic traveling wave solutions; when $h$ varies from 0 to $h_{2}$, these periodic traveling wave solutions will gradually lose their smoothness, and evolve from smooth periodic traveling waves to periodic cusp traveling waves (see Figure 4 (4-5)).

f). If $r>0, n_{1}=1,(p, q) \in C_{4}$; then when $h \in\left(0, h_{2}\right)$ in (1.7), Equation (1.3) has a family of uncountably infinite many periodic traveling wave solutions; when $h$ varies from 0 to $h_{2}$, these periodic traveling wave solutions will gradually lose their smoothness, and evolve from smooth periodic traveling waves to periodic cusp traveling waves (see Figure 4 (4-9)).

2). Suppose that ,then when $h \in\left(0, h_{1}\right)$ in (1.7), Equation (1.3) has two family of uncountably infinite many periodic traveling wave solutions; when $h$ varies from 0 to $h_{1}$, these periodic traveling wave solutions will gradually lose their smoothness, and evolve from smooth periodic traveling waves to periodic cusp traveling waves (see Figure 2 (2-6)).Parallelling to Figure 2 (2-6), we can see the periodic travelling wave solutions implied in Figure 3 (3-6) and Figure 6 (6-2) have the same characters.

3). Suppose that $m-n=2 l-1, n=2 n_{1}+1, l, n_{1} \in Z^{+}$.

g). If $r<0, n_{1}=1,(p, q) \in D_{2} \cup D_{4}$; then when $h \in\left(0, h_{2}\right)$ in (1.7), Equation (1.3) has a family of uncountably infinite many periodic traveling wave solutions; when $h$ varies from 0 to $h_{2}$, these periodic traveling wave solutions will gradually lose their smoothness, and evolve from smooth periodic traveling waves to periodic cusp traveling waves (see Figure 5 (5-6)).

h). If $r<0, n_{1} \geq 2,(p, q) \in D_{2}$; then when $h \in\left(0, h_{1}\right)$ in (1.7), Equation (1.3) has a family of uncountably infinite many periodic traveling wave solutions; when $h$ varies from 0 to $h_{1}$, these periodic traveling wave solutions will gradually lose their smoothness, and evolve from smooth periodic traveling waves to periodic cusp traveling waves (see Figure 5 (5-7)).

i). If $r<0, n_{1} \geq 2,(p, q) \in D_{3} \cup D_{4}$, then when $h \in\left(h_{1}, 0\right)$ in (1.7), Equation (1.3) has a family of uncountably infinite many periodic traveling wave solutions; when $h$ varies from $h_{1}$ to 0 , these periodic traveling wave solutions will gradually lose their smoothness, and evolve from smooth periodic traveling waves to periodic cusp traveling waves (see Figure 5 (5-3)).

j). If $r<0, n_{1} \geq 2,(p, q) \in D_{3} \cup D_{4}$, then when $h \in\left(0, h_{2}\right)$ in (1.7), Equation (1.3) has a family of uncountably infinite many periodic traveling wave solutions; when $h$ varies from 0 to $h_{2}$, these periodic traveling wave solutions will gradually lose their smoothness, and evolve from smooth periodic traveling waves to periodic cusp traveling waves (see Figure 5 (5-5)).

4). Suppose that $n=2 n_{1}+1, l, n_{1} \in Z^{+}, q=0$.

k). If $r<0, n_{1} \geq 2, p>0$, then when $h \in\left(h_{1}, 0\right)$ in (1.7), Equation (1.3) has a family of uncountably infinite many periodic traveling wave solutions; when $h$ varies from $h_{1}$ to 0 , these periodic traveling wave solutions will gradually lose their smoothness, and evolve from smooth periodic traveling waves to periodic cusp traveling waves (see Figure 5 (5-5)).

Equation (1.3) has one family of uncountably infinite many periodic traveling wave solutions; when $h$ varies from 0 to $h_{1}$, these periodic travelling wave solutions will gradually lose their smoothness, and evolve from smooth periodic travelling waves to periodic cusp travelling waves (see Figure 7 (7-4)).

\section{Acknowledgements}

This work is supported by t NNSF of China $(11061010,11161013)$. The authors are grateful for this financial support. 


\section{References}

[1] Song, M. and Shao, S.G. (2010) Exact Solitary Wave Solutions of the Generalized (2+1) Dimensional Boussinesq Equation. Applied Mathematics and Computation, 217, 3557-3563. http://dx.doi.org/10.1016/j.amc.2010.09.030

[2] Chen, H.T. and Zhang, H.Q. (2004) New Double Periodic and Multiple Soliton Solutions of Thegeneralized (2 + 1)-Dimensional Boussinesq Equation. Chaos, Solitons \& Fractals, 20, 765-769. http://dx.doi.org/10.1016/j.chaos.2003.08.006

[3] Li, J.B. (2008) Bifurcation of Traveling Wave Solutions for Two Types Boussinesq Equations. Science China Press, 38, 1221-1234. http://dx.doi.org/10.1007/s11426-008-0129-x

[4] Tang, S.Q., Xiao, Y.X. and Wang, Z.J. (2009) Travelling Wave Solutions for a Class of Nonlinear Fourth Order Variant of a Generalized Camassa-Holm equation. Applied Mathematics and Computation, 210, 39-47. http://dx.doi.org/10.1016/j.amc.2008.10.041

[5] Rong, J.H., Tang, S.Q. and Huang, W.T. (2010) Bifurcation of Traveling Wave Solutions for a Class of Nonlinear Fourth Order Variant of a Generalized Camassa-Holm equation. Communications in Nonlinear Science and Numerical Simulation, 15, 3402-3417. http://dx.doi.org/10.1016/j.cnsns.2009.12.027

[6] Tang, S.Q. and Huang, W.T. (2008) Bifurcations of Travelling Wave Solutions for the K(n,-n,2n) Equations. Applied Mathematics and Computation, 203, 39-49. http://dx.doi.org/10.1016/j.amc.2008.01.036

[7] Li, J.B. and Liu, Z.R. (2002) Travelling Wave Solutions for a Class of Nonlinear Dispersive Equations. Chinese Annals of Mathematics, Series B, 23, 397-418. http://dx.doi.org/10.1142/S0252959902000365

[8] Li, J.B. and Liu, Z.R. (2000) Smooth and Non-Smooth Travelling Waves in a Nonlinearly Dispersive Equation. Applied Mathematical Modelling, 25, 41-56. http://dx.doi.org/10.1016/S0307-904X(00)00031-7

[9] Bibikov, Y.N. (1979) Local Theory of Nonlinear Analytic Ordinary Differential Equations. Lecture Notes in Mathematics, Vol. 702. Springer-Verlag, New York.

[10] Wang, Z.J. and Tang. S.Q. (2009) Bifurcation of Travelling Wave Solutions for the Generalizedzk Equations. Communications in Nonlinear Science and Numerical Simulation, 14, 2018-2024. http://dx.doi.org/10.1016/j.cnsns.2008.06.026

[11] Takahashi, M. (2003) Bifurcations of Ordinary Differential Equations of Clairaut Type. Journal of Differential Equations, 19, 579-599. http://dx.doi.org/10.1016/S0022-0396(02)00198-5 